\title{
A New Kind of Laser Microphone Using High Sensitivity Pulsed Laser Vibrometer
}

\author{
Chen-Chia Wang, Sudhir Trivedi, and Feng Jin \\ Brimrose Corp. of America, 7720 Belair Road, Baltimore, MD 21236 \\ E-mail:ccwang@Brimrose.com \\ V. Swaminathan \\ US Army, RDECOM-ARDEC, Picatinny, NJ 07806-5000 \\ Narasimha S. Prasad \\ NASA Langley Research Center, Mail Stop 468, Hampton, VA 23681
}

\begin{abstract}
We demonstrate experimentally a new kind of laser microphone using a highly sensitive pulsed laser vibrometer. By using the photo-electromotive-force (photo-EMF) sensors, we present data indicating the real-time detection of surface displacements as small as $4 \mathrm{pm}$. (C)2007 Optical Society of America OCIS codes: (120.7280) Vibration analysis (090.2880) Holographic interferometry (170.3890) Medical optics instrumentation
\end{abstract}

Acoustics is a vibrant field with numerous important applications ranging from seismic studies, laser ultrasonics, and photoacoustic detection of molecules. In a typical application, a listening device like microphone is generally used to monitor the acoustic signatures emitted by the target under surveillance. Characteristics of the detected acoustic signals, including its temporal and spectral features, offer invaluable information about, for example, the depository conditions of natural resources, the presence of mechanical defects, and also the presence of specific molecules of interest in a variety of applications.

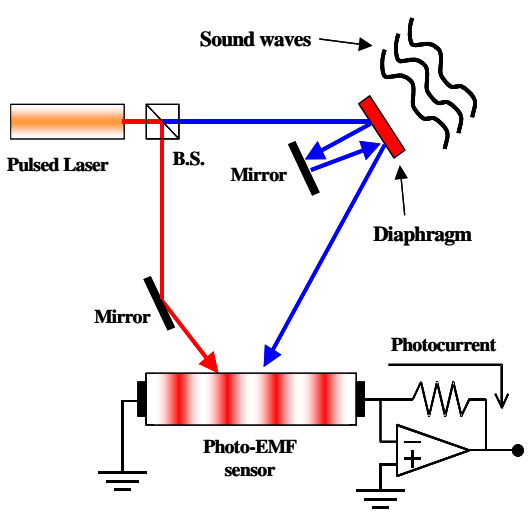

Fig. 1. Schematic of a two-bounce PPLV laser microphone.

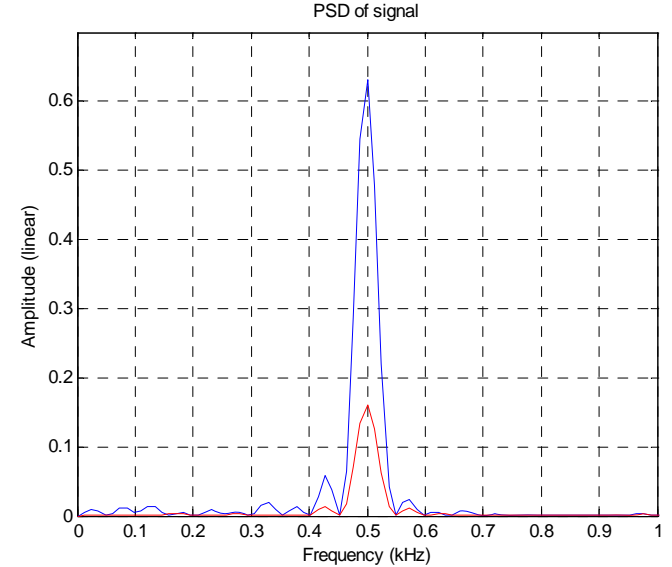

Fig. 2. Power spectra of a vibrating mirror detected by single- (red) and doublebounce (blue) PPLV laser microphone. The mirror was oscillating sinusoidally at $500 \mathrm{~Hz}$ and with the displacement of $3.2 \mathrm{~nm}$.

We recently developed a pulsed laser vibrometer based on the combination of photo-EMF sensors and pulsed light sources, offering an excellent sensitivity of $75 \mathrm{pm}$ in surface displacement detection from optically rough surfaces which produce abundant speckles in the light beams [1]. If the target surface is optically flat and mirror-like, however, the photo-EMF pulsed laser vibrometer (PPLV) can be configured into a laser microphone to detect acoustic signatures being transmitted through an intermediate media like air. Furthermore, the sensitivity in surface displacement detection of the PPLV laser microphone can be enhanced by orders of magnitude by configuring the 
PPLV into multi-bounce laser microphone, as indicated schematically in Figure 1. In such a PPLV laser microphone, the probe light beam is directed onto the diaphragm responsible for sensing the incoming pressure waves. Rather than being sent directly to the photo-EMF sensor for deciphering as in conventional PPLV systems, the back-reflected probe light beam is sent back to the vibrating diaphragm for a number of times/bounces before finally being collected and sent to the photo-EMF sensor for information retrieval. As a result, the total amount of phase modulations suffered by the probe light beam is given by $\phi(t)=\Sigma_{\mathrm{n}} \Delta \sin \left(\omega \mathrm{t}+\mathrm{n} \phi_{0}\right)$, where a sinusoidal pressure wave with the angular frequency $\omega$ is assumed. The number of bounces from the vibrating diaphragm is given by $(n+1)$ where $n=0,1,2 \ldots \ldots$ and $\phi_{0}=\omega \times 2 \mathrm{~L} / \mathrm{c}$ is the additional phase changed experienced by the probe light beam upon its one bounce between the diaphragm and the reflective mirror. Note that $\mathrm{L}$ is the distance between the diaphragm and the bouncing mirror, $\mathrm{c}$ is the speed of light, and $\Delta=2 \pi d / \lambda$ is the maximal phase change due to the displacement $d$ of the diaphragm. $\lambda$ is the wavelength of light. It can be seen readily that if the additional phase shifts $\phi_{0}$ are negligible due to, for example, limited number of bounces or the separation between the diaphragm and the reflective mirror $\mathrm{L}$ is small (relative to the laser pulse width), the total phase shift suffered by the interrogating light beam becomes $\phi(t) \sim \mathrm{N} \times \Delta \sin (\omega \mathrm{t})$ where $\mathrm{N}$ is the total number of bounces from the vibrating diaphragm. That is, under certain conditions, the displacement of the diaphragm can be amplified by the multi-bounce configuration of the PPLV which in turn leads to enhancements in output signal strength as well as the resolution in surface vibration detection. Figure 2 shows an example of the enhanced detection sensitivity of the PPLV laser microphone when it was used to monitor a mirror oscillating sinusoidally at $500 \mathrm{~Hz}$ and with the displacement of 3.2 $\mathrm{nm}$. It is obvious from Figure 2 that the signal strength produced by the double-bounce PPLV laser microphone (blue trace) is enhanced compared to that obtained by the single-bounce PPLV laser microphone (red trace), by a factor of $2^{2}=4$, to be exact. Figure 2 demonstrates the validity of the PPLV laser microphone and points out the route for achieving further improvements in the detection sensitivity for surface vibration monitoring. Indeed, Figure 3 shows the vibration spectrum detected by a multiple-bounce PPLV laser microphone when the surface was vibrating with $500 \mathrm{~Hz}$ in frequency and the displacement of merely $40 \mathrm{pm}$. It is clear from Figure 3 that the detected spectral peak elevates above its adjacent noise floor by a factor of roughly 100 , leading us to believe that, as is, the multi-bounce PPLV laser microphone can decipher surface vibrations with the displacement value of approximately $4 \mathrm{pm}$. Note that the time window width used to collect Figure 3 was approximately $1.8 \mathrm{sec}$. The exact number of bounces involved in Figure 3 is unknown due to limitations of the experimental setup but is estimated to be slightly higher than 20 bounces. Note that the smallest displacement we can produce reliably in the laboratory is currently limited to $40 \mathrm{pm}$.

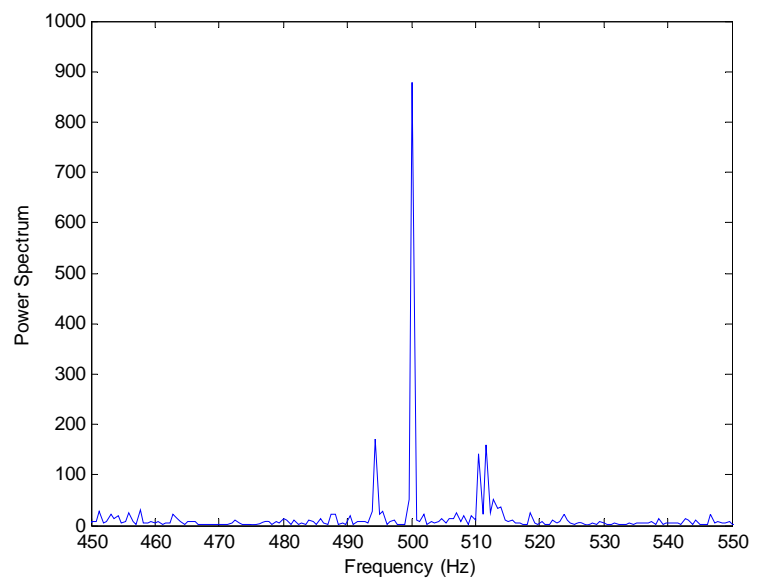

Fig. 3. Vibration spectrum of a mirror oscillating sinusoidally at $500 \mathrm{~Hz}$ and with the displacement of $40 \mathrm{pm}$. The spectrum was detected by a PPLV laser microphone with an estimated 20+ bounces from the vibrating mirror.

\section{References}

[1] P. Rodriguez, S. Trivedi, F. Jin, J. Lorenzo, C.-C. Wang, Z. Chen, J. Khurgin, B. Libbey, and J. Habersat, Technical Digest, CLEO (2006). 